Running Head: ASSESSING SLEEP-RELATED ATTITUDES WITH THE IAT

\title{
Assessing sleep-related attitudes with the implicit association test: \\ a prospective study in young adults
}

Carmen Peuters ${ }^{1,2}$, Jamie Cummins ${ }^{1}$, Ann Vandendriessche ${ }^{3}$, Ann DeSmet ${ }^{4,5}$ \& Geert Crombez ${ }^{1}$

${ }^{1}$ Department of Experimental-Clinical and Health Psychology, Ghent University, Belgium

${ }^{2}$ Department of Movement and Sports Sciences, Ghent University, Belgium

${ }^{3}$ Department of Public Health and Primary Care, Ghent University, Belgium

${ }^{4}$ Department of Communication Studies, University of Antwerp, Belgium

${ }^{5}$ Faculty of Psychological and Educational Sciences, Université libre de Bruxelles, Belgium

Correspondence. carmen.peuters@ugent.be, Henri Dunantlaan 2, 9000 Ghent, Belgium, tel. +3292646321 .

Funding information. This research was conducted with the support of the FWO Grant G043319N to Geert Crombez and Ilse De Bourdeaudhuij and a Grant for the development and evaluation of the method MOV-E-STAR of the Flemish Government to Ghent University.

Conflict of interests. The authors declare no conflict of interest.

Author contributions. Study concept and design: CP, JC, AD and GC. Measurement development: all authors. Data collection and statistical analysis: CP and JC. Drafting the manuscript: $\mathrm{CP}$ in consultation with JC and GC. Discussion and revisions of the manuscript: all authors.

Total number of words: 4357

Total number of references: 40 


\begin{abstract}
The measurement of automatic attitudes towards sleep, in addition to reflective self-reports, might improve our ability to predict and explain sleep-hindering practices. Two types of implicit association tests (IATs), a sleep-related evaluations IAT and a sleep-related selfidentity IAT, were developed to evaluate their efficacy for assessing automatic sleep-related attitudes. In addition, also a speeded self-report measure of sleep evaluations was explored as a means to assess automatic sleep-related attitudes. The study included 136 young adults (age = $21.70 \pm 2.22,43 \%$ female). At baseline, the two IATs, the speeded self-report, and standard self-reports of sleep determinants (reflective attitudes, self-efficacy, intention and action planning for sleep-promoting behaviour), sleep hygiene practices, sleep quality and sleep duration were assessed. All variables except for the sleep determinants were assessed again at two-week follow-up. Results demonstrated good reliability of the two IAT-versions, but both IATs were unrelated to the speeded self-report, the sleep determinants, sleep practices, sleep quality or sleep duration. The speeded self-report correlated significantly with the standard selfreports of sleep determinants. Baseline scores on the IATs or speeded self-report did not predict sleep hygiene practices, sleep duration or sleep quality at follow-up. The findings indicate that sleep-related IATs might not be suited to assess automatic sleep-related attitudes. Further investigation is needed to determine whether speeded self-reports are valid measures of automatic attitudes. Moreover, more empirical research is required to clarify the role of automatic processes for sleep hygiene behaviours.
\end{abstract}

Keywords: implicit attitudes, indirect measures, sleep promotion, measurement, theory of planned behaviour, health action process approach 


\section{INTRODUCTION}

Lack of adequate sleep is an ongoing public health problem (Hafner et al., 2017; Matricciani et al., 2017). Young adults often experience inadequate sleep and poor sleep quality, increasing their risk for short- and long-term detrimental health effects (Bruce et al., 2017; Itani et al., 2017). Sleep can be improved by changing sleep hygiene practices, such as going to bed on time and not taking electronic devices to bed (Irish et al., 2015). Notably, attitudes are one of the determinants in explaining and predicting health-related behaviour across different domains (Sheeran et al., 2016). In relation to sleep, however, not much research is available on sleeprelated attitudes, and findings on their predictive utility for sleep behaviours are mixed (Kor \& Mullan, 2011; Tagler et al., 2017).

Most research on sleep attitudes has used self-report measures of attitudes, which typically reflect participants' deliberated responses (Murawski et al., 2019; Peach et al., 2018; Tagler et al., 2017). However, attitudes can also be expressed automatically (i.e., without the awareness and/or intention of the participant to express this attitude; Greenwald \& Banaji, 1995; Peters \& Slovic, 2007). Reflective attitudes mostly predict behaviours which involve deliberation, whereas automatic attitudes are most likely to predict behaviours which occur automatically (Dessel et al., 2020, Moors \& de Houwer, 2006). This is particularly relevant for sleep hygiene behaviours, as many can be considered as habitual (e.g., scrolling on the smartphone before bed; Loft, 2012; Rebar et al., 2020). As such, automatic attitudes might be superior to reflective attitudes in predicting these habitual sleep-hindering practices.

To assess automatic attitudes, psychologists often use implicit measures (Houwer, 2006) which typically measure participants' reaction times while categorising attitude-related stimuli (Albarracin \& Johnson, 2018). The most commonly used implicit measure is the Implicit Association Test (IAT; Greenwald et al., 1998). The IAT generally comes in the form of an evaluations IAT, where attitude-related stimuli are categorised alongside positive and negative 
category stimuli. Different category stimuli can be used to tap into other attitude-related dimensions. The self-identity IAT (Greenwald et al., 2002) uses self-and other-related category stimuli to capture the extent to which individuals automatically identify themselves with the behaviour (e.g., sleep-promoting practices). The procedure of both types of IAT is illustrated in Figure 1.

Of note, the IAT assesses automatic attitudes indirectly (i.e., does not ask directly about the attitude in question). However, it may well be that automatic attitudes can also be probed directly, this is, by getting participants to respond very quickly on a self-report measure (Ranganath et al., 2008). If proven valid, this approach could be preferred to using an IAT, given the easier administration and completion of such a task. It is therefore worthwhile to also explore the validity of a speeded self-report measure, in addition to an evaluations IAT and a self-identity IAT, as a means to capture automatic sleep-related attitudes.

\subsection{This study}

This study examined whether a sleep-related evaluations or self-identity IAT predict sleeprelated behaviours and outcomes. In particular, we tested the efficacy of the sleep IATs in predicting sleep-promoting behaviour (i.e., sleep hygiene), sleep quality, and sleep duration two weeks later. To examine validity, we furthermore investigated the association of the sleep IATs with other sleep determinants: self-reported reflective attitudes (cognitive and affective evaluations, outcome expectancies), perceived self-efficacy, behavioural intentions, and action planning (Schwarzer, 2008). Finally, these two research questions were also investigated using a speeded self-report measure of sleep evaluations.

*** insert approximately here Figure $1 * * *$ 


\section{METHODS}

\subsection{Participants}

Participants were recruited via Prolific Academic, an online research platform which has been shown to produce high-quality data (Peer et al., 2017). To validate the sleep IAT measures in both English and Dutch, the study was set up in both languages. The sample included young adults aged between 18 and 26 years old, with no diagnosed sleeping problems. Further inclusion criteria were a 90\% approval rating on Prolific and proficiency in either Dutch (Dutch version) or English (English version). Participant drop-out and exclusions are presented in Figure 2. The final sample consisted of 136 participants (mean age $=21 \pm 2$ years; $43 \%$ female, $56 \%$ male, $1 \%$ identifying outside of gender binary) of whom most (93\%) had obtained a secondary education diploma or higher.

*** insert approximately here Figure $2 * * *$

\subsection{Procedure}

Data were collected in September 2020 (between the first and second wave of the COVID-19 pandemic in Europe). Participants first provided consent and demographic information. They then completed the two IATs (administered via lab.js) offered in a random order. Thereafter, participants completed the speeded self-report, the sleep behaviour determinants, and the sleep behaviour outcomes (assessed via LimeSurvey). Two weeks later, participants were invited through Prolific to complete a follow-up assessment. At this followup, participants completed one IAT (the first of the two they completed at baseline; this was done to reduce participant burden at follow-up) and the sleep behaviour outcomes. Participants were thanked and received $£ 5$ as an incentive for their participation. The study instructions and surveys of both assessment points can be found in Supplementary Material 1. The study was 
approved by the Ethics Committee of the Faculty of Psychology and Educational Sciences of Ghent University (registration number: 2020/53) and preregistered at https://osf.io/nfstb.

\subsection{Measures}

\subsubsection{Development and description of the IATs}

We developed the sleep evaluations IAT and the sleep self-identity IAT based on the standard IAT procedure (Greenwald et al., 1998). Both IATs consisted of seven blocks as illustrated in Figure 1. On all trials in all blocks, upon presentation of a stimulus in the centre of the screen, participants had to categorize the stimulus to either a category presented on the top right of the screen (by pressing the right response key, i.e., "M" on the computer keyboard for the Dutch version, assuming an AZERTY keyboard; and "L" for the English version, assuming a QWERTY keyboard) or to a category on the top left of the screen (by pressing the left response key, i.e., "Q" for the Dutch version and "A" for the English version). All stimuli were presented until a response was detected. Incorrect responses (i.e., a wrong categorization) resulted in an error message. For both IATs, participants started with a learning phase of two blocks (firstly an attribute categorization practice block of 20 trials, and then a target categorization practice block of 20 trials), followed by two combined blocks in which the targets and attributes appeared in random order on alternating trials (the first 20 trials, the second 40 trials). The fifth block was again a target categorization practice block with reversed response assignment (20 trials). The final two blocks were another pair of combined blocks, with the reversed assignment for the target categories (the first 20 trials, the second 40 trials).

\section{Evaluations IAT}

The sleep evaluations IAT was based upon the standard procedures for an IAT with an evaluative (pleasant vs. unpleasant) attribute dimension (Greenwald et al., 1998). The stimuli for the attributes were the pleasant-relevant words "peace", "happy", "kind", and "love"; and the unpleasant-relevant words "war", "unhappy", "unkind", and "hate" (Greenwald et al., 
1998). For the target stimuli, the authors worked with words describing practices that are either 'sleep-promoting' or 'sleep-hindering'. To develop the specific target stimuli, 6 sleeppromoting and 6 sleep-hindering practices were pretested in a sample of 15 young adults on (a) relevance, (b) comprehensibility, and (c) strength of categorisation as either sleep-promoting or sleep-hindering (see Supplementary Material 2). Based on these results, the following items were considered valid target stimuli for an IAT with sleep-promoting versus sleep-hindering practices as target concepts: "active day, calm evening", "going to sleep on time", "darken your bedroom", and "going to sleep at the same time every day" for 'sleep-promoting'; and "gaming right before bed", "using smartphone right before sleep", "keep watching videos", and "caffeinated drinks in the evening" for 'sleep-hindering'.

\section{Self-identity IAT}

The self-identity IAT was based upon the standard procedures for an IAT with a self-concept (me vs. other) attribute dimension (Greenwald \& Farnham, 2000). The attribute stimuli for the self-identity IAT were the pronouns "I", "me", "mine" and "self" for the 'me'-category; and "they", "them", "theirs" and "other" for the 'other'-category (based on (Greenwald \& Farnham, 2000). The target concepts were, as in the evaluations IAT, sleep-promoting versus sleephindering practices. The exact same target stimuli were used as described for the evaluations IAT.

\subsubsection{Self-reported sleep behaviour determinants}

Speeded self-reported attitudes towards sleep-promoting behaviour

A speeded self-report measure of evaluations of sleep-promoting behaviour was constructed based on Ranganath and colleagues (2008). Before proceeding to the items, participants were instructed to give their honest response and to do this as spontaneously and as quickly as possible. After clicking 'next', the items appeared repeating the instruction 'Answer this question as quickly as possible!'. We used two items from a previous study (Vandendriessche 
et al., 2020), being "I believe it is important to go to sleep on time" and "I believe it is important for my sleep not to take any devices (smartphone, tablet, or laptop) to bed". Answer options ranged from 1 (completely disagree) to 7 (completely agree). There was no time limit for answering. The reaction time for each individual item was not recorded. A mean was calculated with higher scores indicating a more positive spontaneous evaluation of sleep-promoting behaviour.

\section{Reflective attitudes towards sleep-promoting behaviour}

Behavioural attitudes refer to the tendency to evaluate behaviour with some degree of favour or disfavour, including (a) positive or negative evaluations of the behaviour as important or a priority, (b) emotions associated with the behaviour (e.g. satisfaction, annoyance, etc.), and (c) outcome expectancies of the behaviour (e.g., optimal functioning, mood, etc.) (Eagly \& Chaiken, 2007; Peach et al., 2018). Following this conceptualisation, a measure of cognitive and affective evaluations and a measure of outcome expectancies were established to capture reflective attitudes towards sleep-promoting behaviour.

The measure of cognitive and affective evaluations of sleep behaviour was based on (Francis et al., 2004). Participants were asked to evaluate four sleep-facilitating and four sleephindering practices (i.e., the same practices as those used as target stimuli in the IATs) on a 7point Likert scale of stressful-relaxing, useless-useful, annoying-fun, and disadvantageousadvantageous. Separate sum scores were calculated for the sleep-facilitating and sleephindering items, with higher scores indicating more positive evaluations of sleep-facilitating or sleep-hindering behaviours.

The measure of outcome expectancies was restricted to the sleep-promoting behaviour 'going to bed on time'. This was done to reduce question burden and keep the questionnaire short ('going to bed on time' implies the exclusion of many sleep-hindering behaviours, such as watching videos late into the night). Three items assessed expected benefits (e.g., "going to 
bed on time gives me more energy during the day"), and four items assessed expected disadvantages (e.g., "going to bed on time gives me less time for relaxation"). The items were constructed after pretesting 12 items on their relevance and comprehensibility (see Supplementary Material 3). Items were scored on a 7-point Likert scale ranging from "completely disagree" to "completely agree". Separate sum scores were calculated for perceived benefits and perceived disadvantages with higher scores indicating stronger outcome expectancies.

\section{Self-efficacy, intention, and action planning for sleep-promoting behaviour}

Next to behavioural attitudes, perceived self-efficacy (i.e., the belief in one's capability to perform the behaviour), behavioural intention (i.e., explicit decisions to act in a certain way), and action planning (i.e., detailed planning of how and under what circumstances the behaviour is to be implemented) have been identified as other important personal determinants of health behaviour (e.g., Schwarzer, 2008). The scope of the measures for self-efficacy and intention was, as for outcome expectancies, also restricted to the behaviour 'going to bed on time'.

Self-efficacy was measured with three items selected from a questionnaire developed by the third author (Vandendriessche et al., 2020) by means of factor analysis on an existing dataset. Items are formulated as "I think I can go to bed on time, even if ..." followed by a potential obstacle to perform the behaviour (e.g. missing certain chat threads on social media). A sum score was calculated with higher scores indicating a greater sense of self-efficacy.

Behavioural intention was measured with the one item "I have the intention to go to sleep on time for the coming two weeks". The item was scored on a 7-point Likert scale ranging from "completely disagree" to "completely agree".

Action planning was measured with four items based on (Schwarzer, 2008) assessing the degree of concrete action plans for reducing smartphone use before bed, setting the exact bedtime, doing a relaxing activity before bed, and not sleeping too long in the weekend. A 7- 
point Likert scale ( $1=$ completely disagree, $7=$ completely agree $)$ was complemented with an additional answer option 'I do not plan to do this' (scored as 0). A sum score was calculated with higher scores indicating greater action planning.

\subsubsection{Self-reported sleep behaviour outcomes}

\section{Sleep-promoting behaviour}

The Sleep Hygiene Index (Mastin et al., 2006) was used to measure sleep hygiene behaviour. The self-report instrument has shown moderate to good psychometric properties in a nonclinical sample (Mastin et al., 2006), and includes 13 items assessing how frequently the participant engaged in the specific behaviours on a scale from 1 (never) to 5 (always). Higher scores indicate more maladaptive sleep hygiene behaviours.

\section{Sleep quality and sleep duration}

Sleep outcomes were assessed with the Pittsburgh Sleep Quality Index (PSQI; Buysse et al., 1989), which is a widely accepted, reliable, and validated measure of sleep quality. The scale consists of 18 self-report items assessing seven aspects of sleep quality over the past two weeks: sleep efficiency, sleep latency, sleep duration, sleep disturbance, subjective sleep quality, use of sleep medication, and daytime dysfunction. Each aspect is captured in a subscale scored from 0 to 3, and altogether sum up into a global PSQI score. The global PSQI score ranges from 0 to 21 , with higher scores indicating worse sleep quality, and was used as a measure of sleep quality. Answers to the $4^{\text {th }}$ question of the PSQI (i.e., "How many hours of actual sleep did you get at night?") in minutes per night were used as a measure of sleep duration.

\subsection{Data analysis}

All data were processed and analysed using R with the tidyverse and sjPlot packages. An $\alpha$ level of .05 was used. Data for both IATs were scored using the IAT D2 score method (see (Greenwald et al., 2003). To test for stability reliability, Pearson correlations were calculated among the baseline and follow-up scores of the evaluations IAT, the self-identity IAT and the 
speeded self-report (i.e. test-retest). A split-half approach was used to examine internal consistency of the two IATs. IATs were split between odd-number and even-number trials, and the correlation between the odd and even scores was calculated. Applying the scoring algorithm to each set of responses, the Pearson correlation between the two resulting scores was computed. Internal consistency of the speeded self-report was examined by calculating the Pearson correlation among the baseline scores on the two items.

To examine the extent to which the participants' IAT scores and speeded self-report scores correlated with their scores on the self-reported sleep determinants and sleep behaviour, a correlations matrix was computed among baseline scores of these variables.

Finally, to examine whether IAT scores predicted self-reported sleep behaviour, a series of linear regressions were performed with sleep hygiene, sleep duration, and sleep quality at follow-up as dependent variables. Separate analyses were conducted for baseline scores on the IATs, the speeded self-report, evaluations, outcome expectancies, self-efficacy, intention and action planning of sleep hygiene behaviour as predictors.

\section{RESULTS}

\subsection{Descriptive data and reliability tests}

Descriptive study data (mean, standard deviation, observed range and scale range) of the predictor and outcome variables are presented in Table 1. All variables were normally distributed based on visual inspection of the distribution of the residuals.

The reliability of the IATs was within the modal range as found in previous research (Lane et al., 2007). The evaluations IAT had a split-half reliability of .72 with $95 \% \mathrm{CI}=[.67$, $.78]$ and a test-retest reliability of $.49[.28, .65]$. The self-identity IAT had a split-half reliability of $.65[.58, .71]$ and a test-retest reliability of $.35[.14, .54]$. The 2 -item speeded self-report had an internal consistency of .64 $[.53, .74]$ and adequate test-retest reliability $(r=.72[.64, .79])$. 


\subsection{Baseline correlations}

Table 1 shows the baseline correlations of the evaluations IAT, the self-identity IAT and the speeded self-report with the sleep determinants and sleep behaviour outcomes.

\subsubsection{IATs}

The IATs were not significantly correlated with the self-reported determinants. The only significant correlation between the IATs and self-report measures was a positive correlation between scores on the evaluations IAT with perceived disadvantages of going to bed on time, $r=.20,95 \%$ CI $[.03, .35], p=.02$. Given the likely high rate of Type I error associated with multiple comparisons, we interpret this as most likely noise.

\subsubsection{Speeded self-report of sleep attitudes}

Higher scores on the speeded self-report were significantly correlated with more positive cognitive and affective evaluations of sleep-facilitating activities $(r=.41,95 \% \mathrm{CI}[.26, .54], p$ $<.001)$, more negative cognitive and affective evaluations of sleep-hindering activities $(\mathrm{r}=$ $.42[-.54,-.26], p<.001$, $)$, stronger perceived benefits of going to bed on time $(r=.42[.27$, $.55], p<.001)$, and higher self-efficacy $(r=.18[.01, .34], p=.04)$, intention $(r=.39[.24, .52]$, $p<.001)$ and action planning $(r=.36[.20, .50], p<.001)$ for going to bed on time.

\subsection{Predicting sleep behaviour}

\subsubsection{IATs and speeded self-report of sleep attitudes}

Baseline scores on the evaluations IAT, the self-identity IAT, or the speeded self-report did not predict sleep hygiene, sleep quality, or sleep duration two weeks later. Regression estimates are presented in Table 2 . 


\subsubsection{Self-reported evaluations, outcome expectancies, self-efficacy, intention and action planning of sleep-promoting behaviour}

Cognitive and affective evaluations of sleep-facilitating activities and outcome expectancies of going to bed on time (i.e. perceived benefits and perceived disadvantages) did not predict selfreported sleep hygiene, sleep quality, or sleep duration two weeks later. Evaluating activities that hinder sleep as nice or useful predicted worse sleep hygiene two weeks later $(B=0.15$, $95 \%$ CI $[0.03,0.27], p=.011)$, but did not predict sleep quality or sleep duration. Greater selfefficacy $(B=-0.10[-0.14,-0.04], p=.001)$, intentions for going to bed on time $(B=-0.06[0.11$, $-0.01], p=.025)$ and action planning for sleep-promoting behaviour $(B=-0.09[-0.15,-0.03]$, $p=.004)$ significantly predicted better sleep hygiene at two-week follow-up. Self-efficacy, intentions and action planning did not predict sleep quality or sleep duration two weeks later.

*** insert approximately here Table $2 * * *$

\section{DISCUSSION}

In this study, we developed an evaluations IAT and self-identity IAT for sleep, and investigated their ability to predict sleep hygiene, quality, and duration two weeks later. We also investigated their cross-sectional associations with self-reported determinants of sleep-promoting behaviour. Although the IATs had been carefully developed and showed good split-half and test-retest reliability, they did not predict any of the sleep outcomes, and were not associated with selfreported sleep determinants.

There are several possible explanations for these results. First, one could argue that people have dual attitudes towards sleep-promoting behaviour. This is, automatic sleep attitudes and reflective sleep attitudes that are independent from each other (Wilson et al., 2000). Considering IATs as measures of the automatic attitudes and self-reports as measures of the 
reflective attitudes, our study's weak correlations between the sleep IATs and the self-reported sleep determinants seem to support this dual attitudes view. Nevertheless, we would expect that automatic sleep attitudes have value in predicting, albeit different aspects of sleep behaviour. This was not the case. The IATs were unable to predict any of the sleep hygiene related outcomes.

Second, the poor performance of the IATs may be due to the inability of our IATs to address the highly individual nature of sleep hygiene behaviours. Specific sleep-related behaviours may be more or less relevant for different individuals, and our method did not reflect this. Such personalisation of the IAT has proven beneficial in other health-related contexts (Velkoff \& Smith, 2020). Future research exploring this area may therefore benefit from a more personalised approach to IAT and outcome assessment.

Third, it may well be that the IAT is simply not a valid measure of automatic attitudes. Previous research has shown that the IAT often failed to correlate with other implicit attitude measures (Karpinski \& Hilton, 2001). Moreover, recent evidence indicates that the IAT is a rather noisy measure as compared to self-reports (Schimmack, 2021). Most likely, the IAT is just a poor measure for our purposes.

This study also explored a speeded self-report as measure of automatic sleep-related attitudes. The speeded self-report had good internal consistency and test-retest reliability, but as with the IATs - did not predict sleep outcomes. In contrast to the IATs, the speeded selfreport correlated significantly with reflective attitudes (cognitive and affective evaluations, and perceived benefits), self-efficacy, intention and action planning of sleep-promoting behaviour. Although the speeded self-report only consisted of two items, our preliminary results suggest a better validity as compared to the IAT. Further studies need to further corroborate this idea and explore to what extent a speeded self-report truly measures automatic sleep-related attitudes. Further investigation is required both in terms of identifying valid items (personalisation as 
discussed above may also prove useful here) and in terms of establishing that the response captured in speeded self-reports can be considered automatic.

A remarkable finding of this study was that also most of the reflective attitudes constructs were unable to predict sleep behaviour. This raises the question whether attitudes are in any way good predictors of sleep behaviour. The Theory of Planned Behaviour assumes that intentions mediate the effects of attitudes on behaviour (Ajzen, 1991; Tagler et al., 2017). Indeed, research on sleep has shown that attitudes predict intentions for sleep behaviour, and intentions in turn predict sleep behaviour (Tagler et al., 2017). However, no mediation analyses have yet been done, and findings regarding the total effect of attitudes on sleep behaviour are mixed. Evidence seems to vary dependent on the measure used to assess sleep behaviour. Sleep hygiene behaviour when measured with the Sleep Hygiene Practice Scale (SHPS; (Lin et al., 2007) has repeatedly been predicted by attitudes (Peach et al., 2018; Ruggiero et al., 2019), whereas measured via the Sleep Hygiene Index (as in this study) it has not (Murawski et al., 2019). The wide range of practices that fall under the general construct of sleep hygiene behaviour (e.g., optimizing the environment, evening habits, sleep routine, etc.) complicate its investigation. Narrowing assessment to single practices could clarify the role of attitudes in predicting sleep behaviours.

Furthermore, it might well be that positive attitudes are insufficient to predict sleep hygiene, and that other determinants such as self-efficacy play an important role. People with positive attitudes towards sleep hygiene, may still not engage in sleep hygiene practice because of a low self-efficacy and/or the presence of an intention-behaviour gap. The findings that self-efficacy and action planning (i.e., a technique to overcome the intention-behaviour gap; Schwarzer, 2008) predicted sleep hygiene, are in line with this view.

Our study has some limitations. First, we opted for a combination of words representing a sleep practice as target stimuli in the IAT, whereas generally, the stimuli used in IATs are 
either images or single words. This was done because some sleep practices (e.g., 'gaming right before bed') are difficult to capture in a single word or image, and each stimulus used in the IAT needs to be unequivocally unambiguous in meaning. The combination of words, however, might have required too much of reflective processing to still capture an automatic response. Relational implicit measures which can more easily accommodate complex information exist, but have not yet been applied in sleep or other health-related areas (Cummins \& de Houwer, 2020). Second, the present study measured sleep behaviour via retrospective self-reports. Diaries asking participants to report on their sleep practice of the previous day, or masked devices registering participants' activity before and during bedtime, might avoid possible retrospective bias. Third, this study was run during the COVID-19 pandemic. The current sleep practices may not be representative for sleep practices in normal times. Fourth, the study included young people who reported not being diagnosed with a sleeping disorder. Results could be different for clinical populations or samples with other characteristics.

In conclusion, the findings demonstrate that sleep-related IATs appear unsuited to assess sleep-related attitudes. Although it has been suggested that sleep hygiene behaviours are likely to be influenced by automatic processes (Rebar et al., 2020), further empirical research is required in order to substantiate this claim, and our findings seemingly contradict this perspective.

\section{REFERENCES}

Ajzen, I. (1991). The theory of planned behavior. Organizational Behavior and Human Decision Processes, 50, 179-211. https://doi.org/10.1016/0749-5978(91)90020-T

Albarracin, D., \& Johnson, B. (2018). The Handbook of Attitudes, Volume 1: Basic Principles (2nd Edition). Routledge. https://doi.org/10.4324/9781315178103

Bruce, E. S., Lunt, L., \& McDonagh, J. E. (2017). Sleep in adolescents and young adults. Clinical Medicine, 17, 424-428. https://doi.org/10.7861/clinmedicine.17-5-424 
Buysse, D. J., Reynolds, C. F., Monk, T. H., Berman, S. R., \& Kupfer, D. J. (1989). The Pittsburgh sleep quality index: A new instrument for psychiatric practice and research. Psychiatry Research, 28, 193-213. https://doi.org/10.1016/0165-1781(89)90047-4

Cummins, J., \& de Houwer, J. (2020). The Shape of Belief: Developing a Mousetracking-Based Relational Implicit Measure. Social Psychological and Personality Science. https://doi.org/10.1177/1948550620978019

Dessel, P. van, Cummins, J., Hughes, S., Kasran, S., Cathelyn, F., \& Moran, T. (2020). Reflecting on 25 years of research using implicit measures: Recommendations for their future use. Social Cognition, $38, \quad$ S223-S242. https://doi.org/https://doi.org/10.1521/soco.2020.38.supp.s223

Eagly, A. H., \& Chaiken, S. (2007). The advantages of an inclusive definition of attitude. Social Cognition, 25, 582-602. https://doi.org/10.1521/soco.2007.25.5.582

Francis, J., Eccles, M. P., Johnston, M., Walker, A. E., Grimshaw, J. M., Foy, R., Kaner, E. F. S., Smith, L., \& Bonetti, D. (2004). Constructing questionnaires based on the theory of planned behaviour: A manual for health services researchers. Centre for Health Services Research, University of Newcastle upon Tyne.

Greenwald, A. G., \& Banaji, M. R. (1995). Implicit Social Cognition: Attitudes, Self-Esteem, and Stereotypes. Psychological Review, 102(1), 4-27. https://doi.org/10.1037/0033$295 X .102 .1 .4$

Greenwald, A. G., \& Farnham, S. D. (2000). Using the implicit association test to measure selfesteem and self-concept. Journal of Personality and Social Psychology, 79, 1022-1038. https://doi.org/10.1037/0022-3514.79.6.1022

Greenwald, A. G., McGhee, D. E., \& Schwartz, J. L. K. (1998). Measuring individual differences in implicit cognition: The implicit association test. Journal of Personality and Social Psychology, 74, 1464-1480. https://doi.org/10.1037/0022-3514.74.6.1464 
Greenwald, A. G., Nosek, B. A., \& Banaji, M. R. (2003). Understanding and Using the Implicit Association Test: I. An Improved Scoring Algorithm. Journal of Personality and Social Psychology, 85, 197-216. https://doi.org/10.1037/0022-3514.85.2.197

Greenwald, A. G., Rudman, L. A., Nosek, B. A., Banaji, M. R., Farnham, S. D., \& Mellott, D. S. (2002). A unified theory of implicit attitudes, stereotypes, self-esteem, and self-concept. Psychological Review, 109, 3-25. https://doi.org/10.1037/0033-295X.109.1.3

Hafner, M., Stepanek, M., Taylor, J., Troxel, W., \& Stolk, C. (2017). Why sleep matters - the economic costs of insufficient sleep: A cross-country comparative analysis. Rand Health Quarterly, 6(4), 11. https://doi.org/10.7249/rr1791

Houwer, J. de. (2006). What are Implicit Measures and Why are We Using Them? In R. W. Wiers \& A. W. Stacy (Eds.), Handbook of Implicit Cognition and Addiction (pp. 11-28). SAGE Publications, Inc. https://doi.org/10.4135/9781412976237.n2

Hussey, I., Hughes, S., \& Nosek, B. (2020). Attitudes, Identities and Individual Differences: A large dataset for investigating relations among implicit and explicit attitudes and identity. Unpublished Manuscript. https://osf.io/pcjwf.

Irish, L. A., Kline, C. E., Gunn, H. E., Buysse, D. J., \& Hall, M. H. (2015). The role of sleep hygiene in promoting public health: A review of empirical evidence. Sleep Medicine Reviews, 22, 23-36. https://doi.org/10.1016/j.smrv.2014.10.001

Itani, O., Jike, M., Watanabe, N., \& Kaneita, Y. (2017). Short sleep duration and health outcomes: a systematic review, meta-analysis, and meta-regression. Sleep Medicine, 32, 246-256. https://doi.org/10.1016/j.sleep.2016.08.006

Karpinski, A., \& Hilton, J. L. (2001). Attitudes and the implicit association test. Journal of Personality and Social Psychology, 81, 774-788. https://doi.org/10.1037/00223514.81.5.774 
Kor, K., \& Mullan, B. A. (2011). Sleep hygiene behaviours: An application of the theory of planned behaviour and the investigation of perceived autonomy support, past behaviour and response inhibition. Psychology and Health, 26, 1208-1224. https://doi.org/10.1080/08870446.2010.551210

Lane, K. A., Banaji, M. R., Nosek, B. A., \& Greenwald, A. G. (2007). Understanding and using the implicit association test: IV: What we know (so far) about the method. In B. Wittenbrink \& N. Schwarz (Eds.), Implicit measures of attitudes: Procedures and controversies (pp. 59-102). The Guilford Press. https://psycnet.apa.org/record/200701388-003

Lin, S. C., Cheng, C. P., Yang, C. M., \& Hsu, S. C. (2007). Psychometric properties of the Sleep Hygiene Practice Scale. 21st Annual Meeting of the American-Professional-SleepSocieties, 30, A262-A262.

Loft, M. (2012). Response to debate on the self-report habit index. Annals of Behavioral Medicine, 44, 141. https://doi.org/10.1007/s12160-012-9361-x

Mastin, D. F., Bryson, J., \& Corwyn, R. (2006). Assessment of sleep hygiene using the sleep hygiene index. Journal of Behavioral Medicine, 29, 223-227. https://doi.org/10.1007/s10865-006-9047-6

Matricciani, L., Bin, Y. S., Lallukka, T., Kronholm, E., Dumuid, D., Paquet, C., \& Olds, T. (2017). Past, present, and future: trends in sleep duration and implications for public health. Sleep Health, 3, 317-323. https://doi.org/10.1016/j.sleh.2017.07.006

Moors, A., \& de Houwer, J. (2006). Automaticity: A theoretical and conceptual analysis. Psychological Bulletin, 132, 297-326. https://doi.org/10.1037/0033-2909.132.2.297

Murawski, B., Plotnikoff, R. C., \& Duncan, M. J. (2019). Development and psychometric testing of an instrument to assess psychosocial determinants of sleep hygiene practice. Journal of Health Psychology. https://doi.org/10.1177/1359105319891524 
Peach, H. D., Gaultney, J. F., \& Ruggiero, A. R. (2018). Direct and Indirect Associations of Sleep Knowledge and Attitudes With Objective and Subjective Sleep Duration and Quality via Sleep Hygiene. Journal of Primary Prevention, 39, 555-570. https://doi.org/10.1007/s10935-018-0526-7

Peer, E., Brandimarte, L., Samat, S., \& Acquisti, A. (2017). Beyond the Turk: Alternative platforms for crowdsourcing behavioral research. Journal of Experimental Social Psychology, 70, 153-163. https://doi.org/10.1016/j.jesp.2017.01.006

Peters, E., \& Slovic, P. (2007). Affective asynchrony and the measurement of the affective attitude component. Cognition and Emotion, 21, 300-329. https://doi.org/10.1080/02699930600911440

Ranganath, K. A., Smith, C. T., \& Nosek, B. A. (2008). Distinguishing automatic and controlled components of attitudes from direct and indirect measurement methods. Journal of Experimental Social Psychology, 44, 386-396. https://doi.org/10.1016/j.jesp.2006.12.008

Rebar, A. L., Reynolds, A. C., Ferguson, S. A., \& Gardner, B. (2020). Accounting for automatic processes in sleep health. Journal of Sleep Research, 29. https://doi.org/10.1111/jsr.12987 Ruggiero, A. R., Peach, H. D., \& Gaultney, J. F. (2019). Association of sleep attitudes with sleep hygiene, duration, and quality: A survey exploration of the moderating effect of age, gender, race, and perceived socioeconomic status. Health Psychology and Behavioral Medicine, 7, 19-44. https://doi.org/10.1080/21642850.2019.1567343

Schimmack, U. (2021). The Implicit Association Test: A Method in Search of a Construct. Perspectives on Psychological Science, 16, 396-414. https://doi.org/10.1177/1745691619863798

Schwarzer, R. (2008). Modeling health behavior change: How to predict and modify the adoption and maintenance of health behaviors. Applied Psychology, 57, 1-29. https://doi.org/10.1111/j.1464-0597.2007.00325.x 
Sheeran, P., Maki, A., Montanaro, E., Avishai-Yitshak, A., Bryan, A., Klein, W. M. P., Miles, E., \& Rothman, A. J. (2016). The impact of changing attitudes, norms, and self-efficacy on health-related intentions and behavior: A meta-analysis. Health Psychology, 35, 11781188. https://doi.org/10.1037/hea0000387

Tagler, M. J., Stanko, K. A., \& Forbey, J. D. (2017). Predicting sleep hygiene: a reasoned action approach. Journal of Applied Social Psychology, 47, 3-12. https://doi.org/10.1111/jasp.12411

Vandendriessche, A., Verloigne, M., Boets, L., Joriskes, J., DeSmet, A., Dhondt, K., \& Deforche, B. (2020). Factoren die samenhangen met slaapgedrag bij adolescenten. JGZ Tijdschrift Voor Jeugdgezondheidszorg, 52, 125-131. https://doi.org/10.1007/s12452$020-00221-8$

Velkoff, E. A., \& Smith, A. R. (2020). Preliminary development of an implicit association test to measure body dissatisfaction and predict disordered eating behaviors. Body Image, 34, 51-58. https://doi.org/10.1016/j.bodyim.2020.05.005

Wilson, T. D., Lindsey, S., \& Schooler, T. Y. (2000). A Model of Dual Attitudes. Psychological Review, 107, 101-126. https://doi.org/10.1037/0033-295X.107.1.101

\section{FIGURE LEGENDS}

Figure 1: Procedure of the sleep implicit association tests (IATs). Stimuli appear in random order in the middle of the computer screen. Participants are instructed to classify as quickly as possible each stimulus into one of two categories (or category pairs, for the combined blocks) presented on the top of the screen, by pressing either a key on the right or on the left of the keyboard. The stimuli in the attribute categorisation blocks are pleasant or unpleasant words (evaluations IAT) or self or other pronouns (self-identity IAT). The stimuli in the target categorisation blocks are sleep-promoting or sleep-hindering practices. In the combined blocks, both categorisation and target stimuli appear. Shorter reaction times for correct categorisation 
as sleep-promoting when paired with pleasant/me than with unpleasant/other, would be indicative of more positive attitudes towards sleep-promoting behaviour.

Figure 2. Flowchart of participant drop-out and exclusions, and make-up of the final sample. Note: ${ }^{1}$ Valid reasons participants gave to exclude their data from the analyses were for example "there were people entering my room" or "I couldn't concentrate well". ${ }^{2}$ IAT screening criteria were based on recommendations from (Greenwald et al., 2003; Hussey et al., 2020): (i) $>=35 \%$ of responses $<300 \mathrm{~ms}$ in any practice block, (ii) $>=25 \%$ of responses $<300 \mathrm{~ms}$ in any critical block, (iii) $>10 \%$ of responses $<300 \mathrm{~ms}$ across all critical blocks, (iv) $>50 \%$ error rate in any given practice block, $(\mathrm{v})>40 \%$ error rate across all practice blocks, (vi) $>40 \%$ error rate in any given critical block, (vii) $>30 \%$ error rate across all critical blocks, and (viii) $>=10 \%$ of responses $>10000 \mathrm{~ms}$ in any given critical block.

\section{SUPPORTING INFORMATION}

Supplementary Material 1, Supplementary Material 2 and Supplementary Material 3 are included as additional supporting information.

\section{DATA AVAILABILITY STATEMENT}

The data that support the findings of this study are available upon request on Open Science Framework at https://osf.io/ubd2h/. 